\title{
Underwater Image Clearance using Dark Channel and FFT Enhancement
}

\author{
Richa Gupta \\ M. Tech. Scholar \\ CSE Dept. \\ ASCT, Bhopal
}

\author{
Zuber Farooqui \\ Asst. Prof \\ CSE Dept. \\ ASCT, Bhopal
}

\begin{abstract}
Due to the absorption and scattering, the clarity and therefore the observation of the depth of field of the image that is obtained by underwater physical phenomenon imaging are going to be reduced. This review paper deals with the ways to enhance underwater image improvement techniques, the process of underwater image captured is critical as a result of the standard of underwater pictures have an effect on and these image leads some serious issues compared to photographs from a clearer setting. plenty of noise happens thanks to low distinction, poor visibility conditions (absorption of natural light), non uniform lighting and small color variations, pepper noise and blur impact within the underwater pictures owing to of these reasons variety of ways are existing to cure these underwater pictures totally different filtering techniques also are obtainable within the literature for process and improvement of underwater pictures one in every of them is image improvement victimization median filter which boosts the image and facilitate to estimate the depth map and improve quality by removing noise particles with the assistance of various techniques, and therefore the alternative is RGB Color Level Stretching have used. This paper proposes AN efficient and quick underwater haze removal technique with quality improvement. This technique involves two phases. The primary section is employed to get rid of underwater haze from a picture that is victimization underwater haze removal technique supported previous data. Second section enhances quality of underwater hazy image improved visibility and noise reduction victimization FFT (Fast Fourier Transformation). This technique is often applied to any style of pictures like RGB Color, gray scale.
\end{abstract}

\section{Keywords}

RGB Color Level, color enhancement, FFT, Dark channel, haze removal.

\section{INTRODUCTION}

Underwater vision is one in all the scientific fields of investigation for researchers. Autonomous Underwater Vehicles (AUV) and Remotely Operated Vehicles (ROV) area unit typically utilized to capture the info like underwater mines, shipwrecks, coral reefs, pipelines and telecommunication cables from the underwater setting. Underwater pictures area unit basically characterized by their poor visibility as a result of lightweight is exponentially attenuated because it travels within the water, and therefore the scenes result poorly contrasted and hazy. Lightweight attenuation limits the visibility distance at regarding twenty meters in clear water and 5 meters or less in murky water. The sunshine attenuation method is caused by absorption and scattering, that influence the performance of underwater imaging systems. Forward scattering typically results in blur of the image options.
On the opposite hand, backscattering typically limits the distinction of the photographs, generating a characteristic veil that superimposes itself on the image and hides the scene. Absorption and scattering effects don't seem to be solely owing to the water itself however conjointly owing to the elements like a dissolved organic matter. The visibility vary will be augmented with artificial illumination of sunshine on the article, however it produces non-uniform of sunshine on the surface of the article and manufacturing a bright spot within the center of the image with poorly lighted space encompassing it. The quantity of sunshine is reduced once we go deeper, colors drop off counting on their wavelengths. The blue color travels across the longest within the water owing to its shortest wavelength. Underwater image suffers from restricted vary visibility, low distinction, non-uniform lighting, blurring, bright artifacts, color diminished and noise

The analysis on underwater image process will be selfaddressed from two completely different points of read like a picture restoration or a picture sweetening methodology $[1,2$, $3]$. The image restoration aims to recover a degraded image employing a model of the degradation Associate of the first image formation; it's basically an inverse drawback. These ways area unit rigorous, however they need several model parameters like attenuation and diffusion coefficients that characterize the water murkiness and might be very variable. Whereas image sweetening uses qualitative subjective criteria to supply a a lot of visually pleasing image and that they don't think about any physical model for the image formation. These varieties of approaches area unit typically easier and quicker than deconvolution ways. Recently, several researchers have developed preprocessing techniques for underwater pictures victimization image sweetening ways. Bazeille et al. [4] propose Associate in formula to pre-process underwater pictures. It reduces underwater perturbations and Prabhakar CJ, Praveen Kumar element Bioinfo Publications improves image quality. The formula is automatic and needs no parameter adjustment. the tactic was used as a preliminary step of edge detection. The lustiness of the tactic was analyzed victimization gradient magnitude histograms and conjointly the criterion utilized by Arnold-Bos et al. [5] was applied. This criterion assumes that well-contrasted and noisefree pictures have a distribution of the gradient magnitude bar graph near exponential, and it attributes a mark from 0 to 1 .

\section{PROBLEM FORMULATION}

This paper covers the image improvement techniques and also the image quality improvement victimization filers, the region lightweight could be a major issue to method below water pictures comes from the poor visibility conditions under the water, scattering of sunshine and light-weight attenuation owing to all the explanations the underwater pictures suffers plenty and have an effect on their visibility and also the distinction that they contain really. Lightweight attenuation 
limits the visible distance, at regarding twenty meters in clear water and five meters or less in muddy or less muddy water.

Use of median filter that has projected by Hung- Yu rule, PieYin Chen[6] that is employed to estimate the transmission of input image the region lightweight is obtained by victimization dark channel previous and accustomed take away the noise like pepper noise, with this technique the noise is removed and therefore the image that has less quantity of noise and additional improved image is achieved however the particular color distinction and fewer sharp image is a smaller amount correct than the first image therefore in future there's a necessity of some technique additionally to enhance the standard of those quite underwater pictures.

\section{RELATED WORK}

Poor visibility under water is a major problem for oceanic applications of computer vision [7,8]. In order to understand the underwater world better, we often using photovoltaic systems to objects under water for imaging [9]. Actually, underwater scenes are usually veiled by the light interaction with the medium: absorption and scattering of the light induce poor contrast, low luminosity and restricted visibility. And turbine the water is, greater the proportion of scattering part is. Thus the data acquired under the sea often suffer of large defaults and have to be preprocessed before any analysis or understanding. In order to get the quality enhancement image, we will compensate for the effect of the attenuation and restore color balance between physical and images. In the literature, a few approaches have been proposed to enhance the underwater image based on physics-based methods. Using the polarization imaging to improve the visibility of underwater color images which are obtained through natural lighting $[9,11,12]$, automatic underwater image preprocessing [13], underwater image improvement by attenuation inversion with quaternions [14], underwater image improvement victimization AN integrated color model [15], comparison and validation of purpose unfold models for imaging in natural waters [16], retinex improvement rule [17] so on.

As light-weight is attenuated once disseminative in water, the clarity of pictures or videos captured underneath water is sometimes degraded to variable degrees. By exploring the distinction in light-weight attenuation between in atmosphere and in water, paper [19] derive a replacement underwater optical model to explain the formation of AN underwater image within the true physical method, then propose an efficient improvement rule with the derived optical model to enhance the perception of underwater pictures or video frames. In our rule, a replacement underwater dark channel springs to estimate the scattering rate, and an efficient methodology is additionally conferred to estimate the background light-weight within the underwater optical model. Experimental results show that our rule will well handle underwater pictures, particularly for sea pictures and people captured from murky waters.

\section{PROPOSED WORK}

Underwater environments typically cause color scatter and color forged throughout photography. Color scatter is caused by haze effects occurring once lightweight returned from objects is absorbed or scattered multiple times by particles within the water. This successively lowers the visibility and brightness of the image. Color forged is caused by the varied attenuation of sunshine in various wavelengths, rendering underwater environments bluish. To handle distortion from color scatter and color forged, this study proposes associate method to recover underwater picture file that mixes a dehazing scheme with wavelength compensation (WCID). Once the space between the objects and therefore the camera was calculable applying dark channel prior, the haze effects from color scatter were removed by the dehazing formula. Next, estimation of the photography scene depth from the residual energy ratios of every wavelength within the background lightweight of the image was performed. per the number of attenuation of every wavelength, reverse compensation was conducted recreate the distortion from color forged.

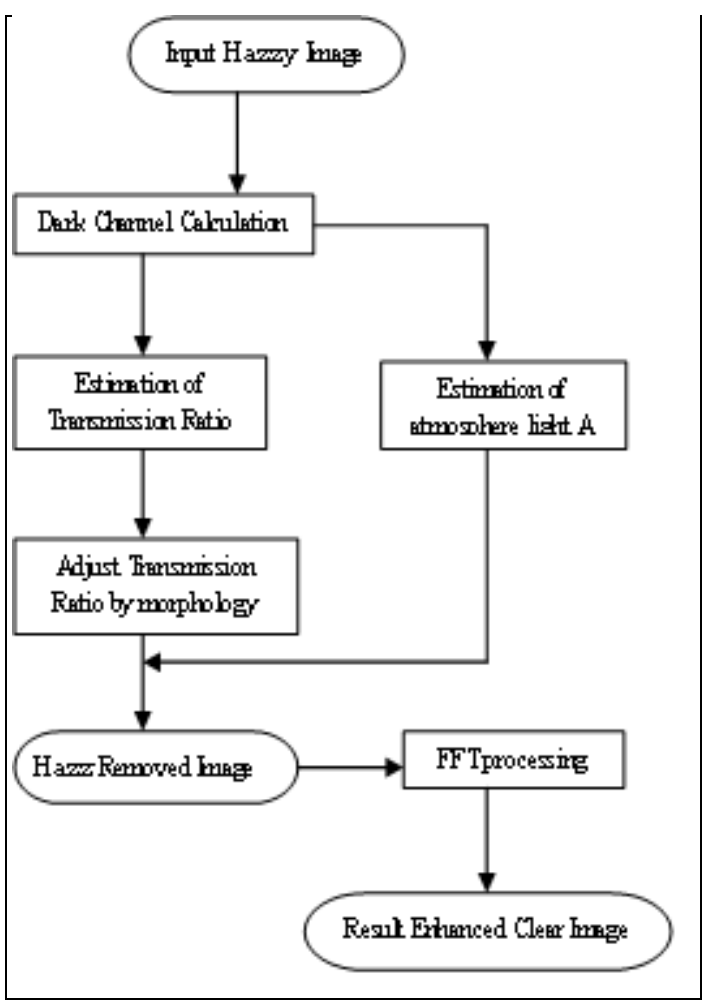

Fig 1: Proposed work flowchart

Overall working of proposed work can be given as flowchart shown in figure no. 1. Figure 2 gives basic block diagram for proposed work architecture.

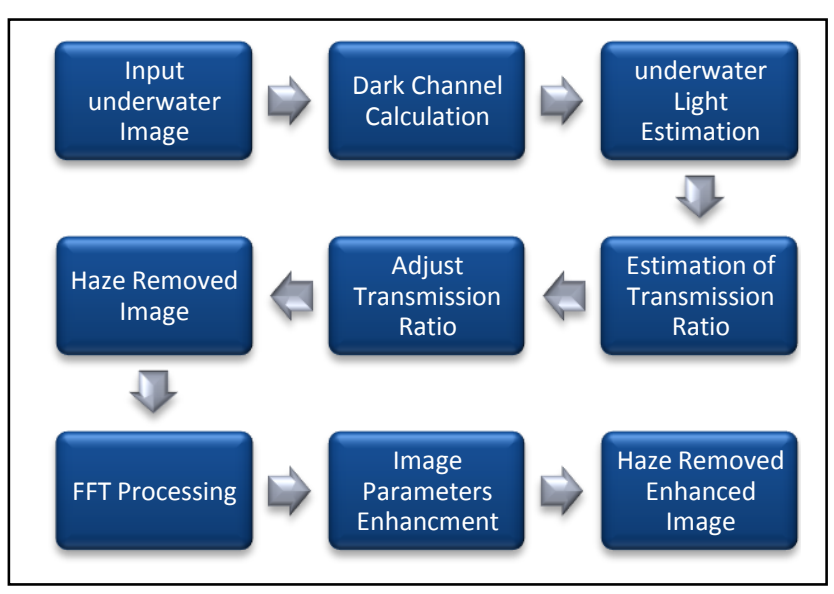

Fig 2: Proposed work architecture

Our proposed work computes image enhancement in two phases. The first phase is used to remove haze from an image. 
Second phase enhance quality of image for improved visibility and noise reduction using FFT (Fast Fourier Transformation).

\subsection{Haze removal based on prior knowledge}

A fast method of hazy image enhancement is given in this section. Firstly, the depth information of the hazy image is extracted according to the hazy image model and the prior knowledge. Secondly, the transmission ratio of the underwater light is estimated and adjusted, and the underwater light $\mathrm{A}$ is also estimated[19]. At last, the Gama adjustment is used to get the final enhancement image. Comparing with other method, the speed is faster than traditional methods.

The model of the hazy image In computation visual the model of hazy image is shown as following, As haze removal Method and Image Enhancement

$$
I(x)=J(x) T(x)+A(1-t(x))
$$

The underwater is homogenous the ratio of transmission is expressed as,

$$
t(x)=e^{-\beta d(x)}
$$

According to the equation, it can be obtained,

$$
t(x)=\frac{\|A-I(x)\|}{\|A-J(x)\|}=\frac{A^{C}-I^{C}(x)}{A^{C}-J^{C}(x)}
$$

Where $\mathrm{C} \varepsilon\{\mathrm{r}, \mathrm{g}, \mathrm{b}\}$, is the color channel index.

The prior knowledge of the dark channel for an image $\mathrm{J}(\mathrm{x})$, we define,

$$
\operatorname{Jdark}(x)=\min (\min (J C(y)))
$$

Except of underwater area, the strength of Jdark is very low and tends to zero if $\mathrm{J}(\mathrm{x})$ is haze-free. So Jdark is called dark channel of $\mathrm{J}(\mathrm{x})$.

1. Estimation of transmission Ratio.

2. The Adjustment of Transmission Ratio by morphological operation.

3. Recovering Source Image $\mathrm{J}(\mathrm{x})$.

4. Estimation underwater light $\mathrm{A}$.

The scheme of fog removal based on prior knowledge overall algorithm given in fig 1 in detail.

\subsection{Quality Enhancement of Haze removal Image}

The FFT is associate economical implementation of DFT and is employed in digital image process. it's wont to convert any image from its abstraction domain to its frequency. Because it is quicker to perform any computation or to use any filter in frequency domain instead of abstraction domain [20].

The calculation of the DFT is incredibly valuable and thus to decrease the value, the FFT came into existence. With the employment of FFT the procedure complications area unit slashed from $\mathrm{N} 2$ to $\log 2 \mathrm{~N}$. as an example, for associate image of size $256 \times 256$ pixels the interval needed is regarding 2 minutes on a general purpose pc. constant machine would take thirty times longer (60 minutes) to calculate the DFT of constant image of size $256 \times 256$.
Fourier remodel converts a picture into its actual and imagined parts that may be a depiction of the image within the frequency domain. Suppose we have a tendency to area unit giving a picture within the style of signaling then variety of frequencies within the frequency domain is same because the number of pixels within the image or abstraction domain. To convert the image into its abstraction domain inverse remodel is applied. The FFT and its inverse of a second image area unit expressed by the equations:

$$
\begin{gathered}
F(x)=\sum_{n=0}^{N-1} f(n) e^{-j 2 \pi\left(x \frac{n}{N}\right)} \\
f(n)=\frac{1}{N} \sum_{n=0}^{N-1} F(x) e^{j 2 \pi\left(x \frac{n}{N}\right)}
\end{gathered}
$$

Where the element at coordinates $(m, n)$ area unit given by $f$ $(m, n)$, the worth of the image within the frequency domain admire the coordinates $\mathrm{x}$ and $\mathrm{y}$ is $\mathrm{F}(\mathrm{x}, \mathrm{y}), \mathrm{M}$ and $\mathrm{N}$ area unit the proportions of the image.

As equation states, execution of this algorithmic program is incredibly pricey. however the good thing about FFT is that it's autonomous, namely, the second remodel is reborn into 2 1D remodels as given in equation (shown solely within the horizontal direction) - one within the horizontal direction followed by the opposite within the vertical direction on the consequence of the horizontal transform. the ultimate result's counterpart of acting the second remodel within the frequency house.

$$
\begin{array}{r}
F(x, y)=\sum_{m=0}^{M-1} \sum_{n=0}^{N-1} f(m, n) e^{-j 2 \pi\left(x \frac{m}{M}+y \frac{n}{N}\right)} \\
f(m, n)=\frac{1}{M N} \sum_{m=0}^{M-1} \sum_{n=0}^{N-1} F(x, y) e^{j 2 \pi\left(x \frac{m}{M}+y \frac{n}{N}\right)}
\end{array}
$$

The FFT that's used on the applying here wants that image dimensions area unit diagrammatical in two's power. a new advantage of FFT is transformation of $\mathrm{N}$ points might categorical as total of N/2 transforms by divide and conquer technique. it's an honest property for scale back calculation effectiveness conjointly take lesser time compare to others.

Fourier Transformation generates results as imaginary number and this numbers have bigger vary than abstraction domain. Thus accuracy represent these values area unit hold on as floats. conjointly vary of constant generated by Fourier transformation is just too giant to displayed on the screen therefore these values area unit reborn to a different formatted as dimension values height*width for decrease the vary and build able to displayed on screen [20].

\section{RESULTS}

The proposed work implemented on matlab 7.10 version and results are compared with existing method given in paper [18]. The projected technique show higher image objects visibility once compare to different strategies. Overall Comparison shown in Figure 3 shows It will be seen that base work has very little or no impact on this type of sea image attributable to the failure of the normal dark channel previous. Projected result has apparent impact for revealing distant details. In Fig. 3 this work at the same time compare our results with those provided by [18]. For the image with fish, our result provides higher improvement for distant objects and background than result well handles distant objects, however there are lot of red parts in their result, particularly within the background. Probably our result's a lot of visually pleasuring. For the image with ship, result unveils the main points solely within the regions on top of the most diagonal of the image and do nothing below the diagonal; result provides no important impact for dehazing and contains evident color 
distortions within the lower left area instead, our result provides better dehazing result within the whole image and unveils a lot of details than result.

Fig. 4 shows a lot of results of our technique. We are able to see that our technique can expose the main points and recover vivid color info even in huge cloudy regions. As an example, the left-hand-most try of pictures in Fig. 4 shows nearly best recovery. Within the high row of Fig. 4, point of the red circle of every input image represents the placement of the background light-weight element found by our technique. Clearly, our technique will realize the precise background lightweight of scenes
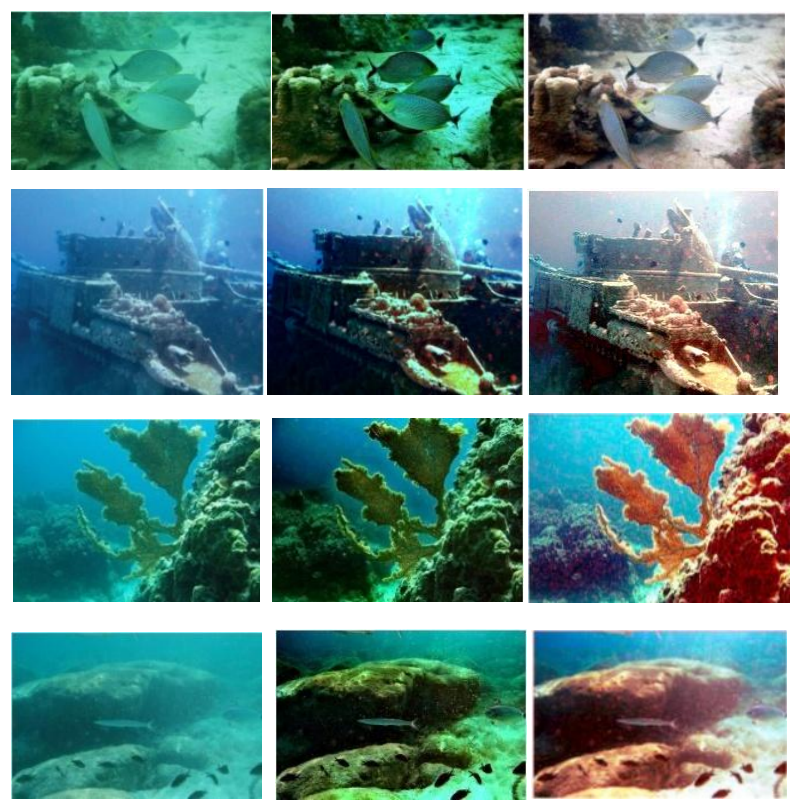

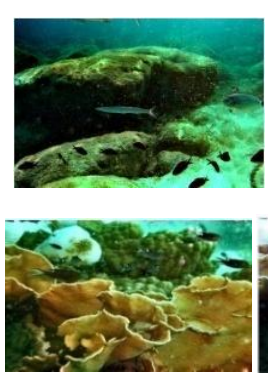

(b)

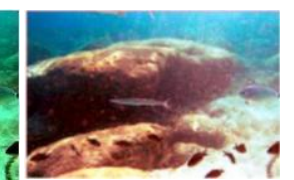

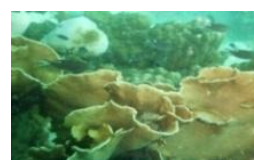

(a)

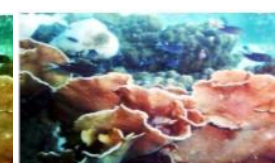

(c)
Fig 3: visual comparisons of the dehazing results of these methods for a underwater image. These methods are Base Paper and the proposed algorithm using image filtering approach. (a) Underwater input image, (b) Base Paper Results, (c) the proposed algorithm results.
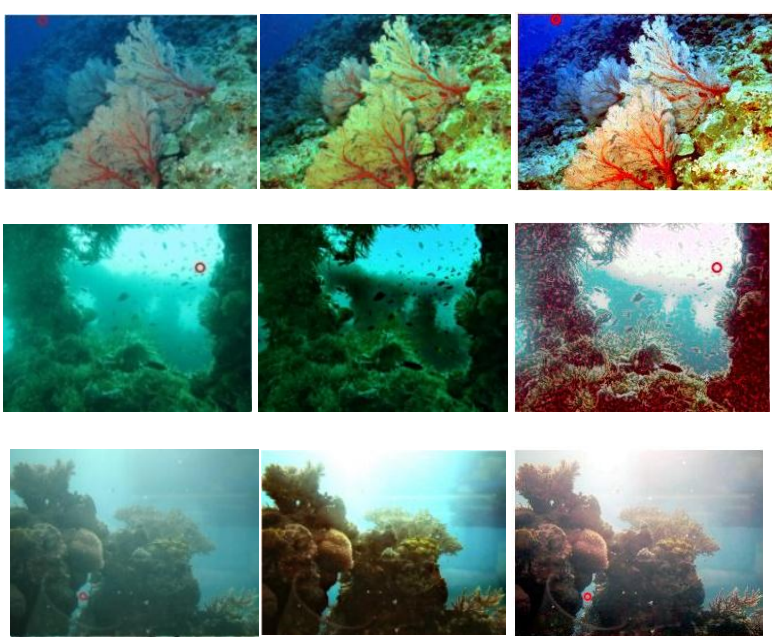

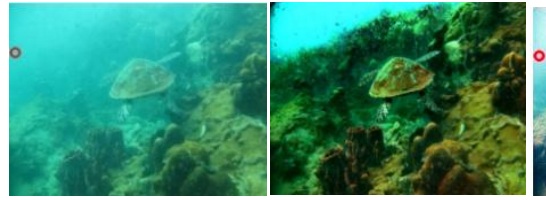

(a) (b)

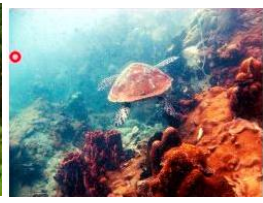

(c)
Fig 4: precise background light of scenes results of proposed method
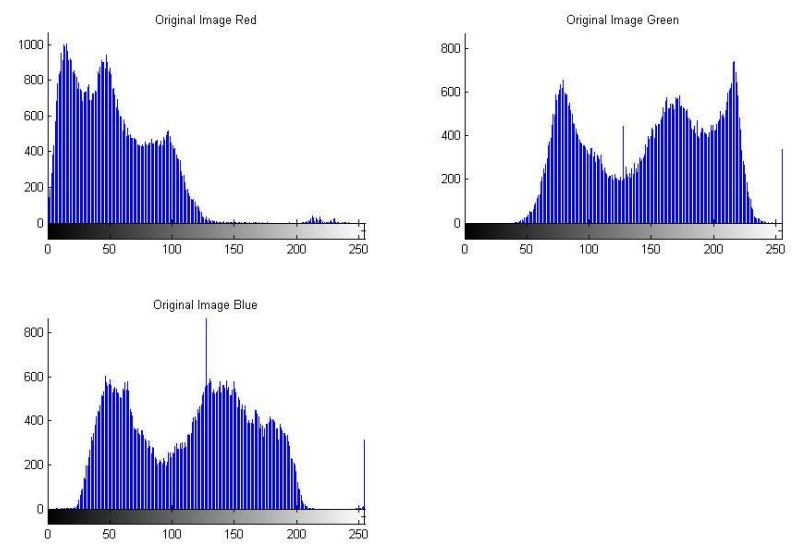

Fig 5: Histogram of input image first of row one is histogram for Red second for Green and last for Blue color histogram of RGB image.
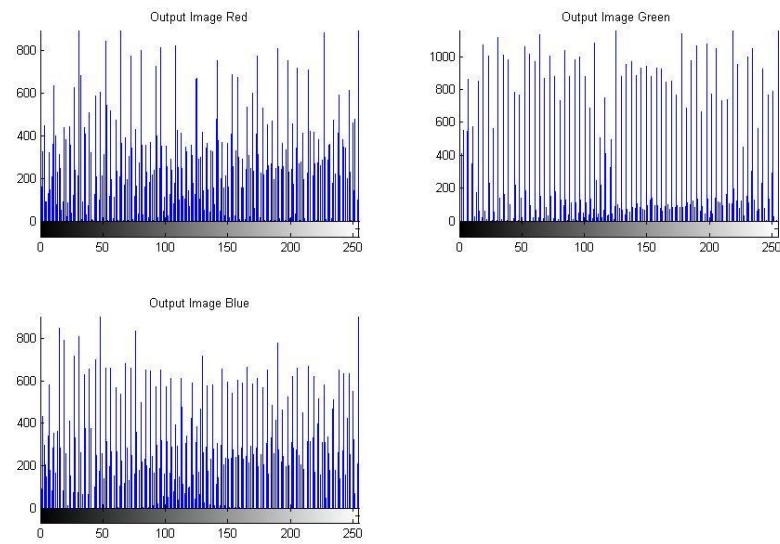

Fig 6: Histogram of output image first of row one is histogram for Red second for Green and last for Blue color histogram of output RGB image

Following figure 5 shows histogram of input hazy image and figure 6 show histogram of output cleared image by observing histogram result may be compare as histogram is properly distributed in output image but in input image histogram is not equalized properly.

\section{CONCLUSIONS}

This work has self-addressed the matter of at the same time removing haze and noise from one image, and has created many novel contributions. the primary is that the adaptation of associate degree existing technique, the dark channel previous, for estimating haze from one clean hazy image to the case of one uncleared hazy image. This paper provides associate degree analysis and comparison of existing image improvement techniques with projected technique. This work additionally represented this progress of image improvement. This paper is proposing a quick haze removal with a top 
quality improvement technique which might be used for next generation traffic and railway image process to get rid of the weather impact from image for more clear visibility and image process. This projected technique has been found more practical than alternative existing technique within the terms of quality and clarity. During this work it compared the results of varied image improvement techniques on completely different grounds like on the pictures of a underwater image in different conditions for different objects found within water, that square measure important aspects for traffic analysis throughout underwater weather. This work has given an efficient and quick underwater image improvement technique for more clear visibility in image or video in real underwater pictures.

\section{REFERENCES}

[1] Raimondo Schettini and Silvia Corchs (2010) Hindawi Publishing Corporation, EURASIP Journal on Advances in Signal processing.

[2] Prabhakar C J and Praveen Kumar P U (2010), in Proceedings of International Conference on Signal and Image Processing, 322-327.

[3] Prabhakar C. J. and Praveen Kumar P. U. (2010) Abstract Proceedings of Seventh Indian Conference on Computer Vision, Graphics and Image Processing (ICVGIP-2010), Chennai, India.

[4] Stephane Bazeille, Isabelle Quidu, Luc Jaulin and JeanPhillipe Malkasse (2006) in Proceedings of the European Conference on Propagation and Systems, Brest, France.

[5] Andreas Arnold-Bos, Jean-Philippe Malkasse and Gilles Kervern (2005) in the Proceedings of the European Conference on Propagation and Systems, Brest, France.

[6] Hung-Yu, Pie-Yin Chen, Chien-Chuan Huang and YaZhu Zhuang second International Conference on Innovations in Bio computing and Applications.

[7] K. Lebart, C. Smith, E. Trucco, and D. M. Lane, "Automatic indexing of underwater survey video: algorithm and benchmarking method,'IEEE J. Ocean. Eng., vol. 28, no. 4, pp. 673-686, Oct. 2003.

[8] J. Yuh and M. West, "Underwater robotics," Adv. Robot., vol. 15, no.5, pp. 609-639, 2001.
[9] JIN W eiqi,WANG Xia,CAO Fengmei, et al. Review of Underwater Opto-electrical Imaging Technology and Equipment(II)[J]. Infrared Technology, 2011, 33(3): 125132.

[10] Schechner Y Y, Karpel N. Recovery of Underwater Visibility and Structure by Polarization Analysis [J]. IEEEJournal of Oceanic Engineering, 2005, 30(3): 570587.

[11] Treibitz $\mathrm{T}$ and Schechner $\mathrm{Y}$ Y. Instant 3Descatter [C]//Proc. IEEE, 2006(2): 1861- 1868.

[12] LI Hailan , WANG Xia , ZHANG Chuntao, et al. The development and analysis of target detection research based on polarization imaging technology $[\mathrm{J}]$. Infrared Technology, 2009, 35(5): 695-700.

[13] Bazeille S,Quidu I,Jaulin L,et al. Automatic Underwater Image Pre-Processing[C]//CMM'06 Caracterisation Du Milieu Marin, 2006:1-18.

[14] Frédéric P,Anne-Sophie Capelle-Laizé and Philippe C. Underwater Image Enhancement by Attenuation Inversion with Quaternions[C]//ICASSP,Proc. IEEE, 2009: 1177-1180.

[15] Kashif I,Rosalina A S,Azam O,et al. Underwater Image Enhancement Using an Integrated Colour Model[J]. IAENG Interna- tional Journal of Computer Science, 2007, 32(2): 239- 244.

[16] Hou W,Gray D J,Weidemann A D,et al. Comparison and validation of point spread models for imaging in natural waters[J]. Optics Express, 2008, 16(13): 9958-9965.

[17] ZHANG Kai,JIN Weiqi,QIU Su,et al. Multi-Scale Retinex Enhancement Algorithm on Luminance Channel of Color Underwater Image[J].Infrared Technolgy,2011, 33(11):630-634.

[18] Haocheng Wen, Yonghong Tian, "Single Underwater Image Enhancement with a New Optical Model", Pages 753-756, IEEE, 2013

[19] Shuai Fang, Rong Deng, "Effective Single Underwater Image Enhancement by Fusion", Journal of Computers, Vol. 8, No. 4, Pages 904-911, April 2013.

[20] Naidu V. P. S., "Multi-resolution image fusion by FFT", International Conference on Image Information Processing (ICIIP), Page: 1-6, IEEE, 2011. 